\title{
O MÉTODO PREVISOR-CORRETOR PRIMAL-DUAL DE PONTOS INTERIORES BARREIRA LOGARÍTMICA CLÁSSI- CA/MODIFICADA COM ESTRATÉGIAS DE PASSO LONGO E CONVERGÊNCIA GLOBAL APLICADO AO PROBLEMA DE FPOR.
}

RICARDO BENTO NOGUEIRA PINHEIRO ${ }^{1}$, ANTONIO ROBERTO BALBO ${ }^{2}$, GERALDO ROBERTO MARTINS DA COSTA ${ }^{1}$.

1. LASEE, Departamento de Engenharia Elétrica, EESC-USP

Av. Trabalhador São-carlense,400 - Centro, CEP:13566-590, São Carlos - SP - Brasil

E-mails: ribenopi@hotmail.com, geraldolsc.usp.br

2. Departamento de Matemática, FC-UNESP

Av. Eng. Luiz Edmundo Carrijo Coube, 14-01, CEP: 17033-360, Bauru-SP-Brasil

E-mail:arbalboefc.unesp.br

\begin{abstract}
In this paper, we present the predictor-corrector primal-dual interior point method with classic/modified logarithmic barrier, long step and global convergence strategies. The main features of the proposed method are: integrated application of the methods of classical and modified logarithmic barrier; proposal to an generalized update strategy of estimators of Lagrange' multipliers; the determination of optimal solutions such that unless it belongs the frontier of the primal feasible region, then these are determined in a non-asymptotic; application of a variant of update sizes primal and dual steps; trajectory modification, correction of the conditioning of the hessian matrix of Lagrangian and global convergence are obtained through a technical variant of the Levenberg-Marquardt and, using the barrier parameter in the calculation of the search directions in the primal and dual predictor procedure. We apply method to the following problems of optimal power flow with reactive taps constants: Electrical System 3, 9, IEEE-14, IEEE-30, IEEE-57, IEEE-118, IEEE-162 and IEEE-300 buses. The results show that this method is robust and efficient for the solution of these problems.
\end{abstract}

Keywords— Reactive Optimal Power Flow, Interior Point Methods, Long Step, Global Convergence.

Resumo- Neste trabalho, apresentamos o método previsor-corretor primal-dual de pontos interiores com barreira logarítmica clássica/modificada com estratégias de passo longo e de convergência global. As principais características do método proposto são: aplicação integrada dos métodos de barreira logarítmica clássica e modificada; proposta de uma estratégia de atualização generalizada dos estimadores dos multiplicadores de Lagrange; determinação de soluções ótimas tais que se pertencerem à fronteira da região de viabilidade primal, então essas são determinadas de modo não-assintótico; aplicação de uma variante da atualização dos tamanhos dos passos primal e dual; modificação de trajetória, correção do condicionamento da matriz hessiana da função lagrangiana e convergência global são obtidas através de uma técnica variante a de Levenberg-Marquardt e; utilização do parâmetro de barreira no cálculo das direções de busca primal e dual no procedimento previsor. Aplicamos o método aos seguintes problemas de fluxo de potência ótimo reativo com taps constantes: sistema elétrico 3, 9, IEEE-14, IEEE-30, IEEE-57, IEEE-118, IEEE-162 e IEEE-300 barras. Os resultados obtidos pelo método mostram que este é robusto e eficiente para a solução destes problemas.

Palavras-chave—Fluxo de Potência Ótimo Reativo, Método de Pontos Interiores, Passo Longo, Convergência Global.

1 O método previsor-corretor primal-dual de pontos interiores com barreira logarítmica clássica/modificada com estratégias de passo longo e de convergência global.

Nesta seção apresentamos o método previsor-corretor primal-dual de pontos interiores com barreira logarítmica clássica/modificada com estratégias de passo longo e de convergência global (MPIBLC/M-PLCG) o qual é uma variante do método primal-dual apresentado em (Pinheiro, 2012). Esse foi desenvolvido para problemas de programação não-linear e não convexa para posterior aplicação em problemas de fluxo de potencia ótimo reativo (FPOR).

Seja o problema de otimização não-linear com restrições de igualdade e de desigualdade de acordo com (1):

$$
\begin{cases}\text { Min } & f(\mathbf{x}) \\ \text { s.a: } & \left\{g_{t}(\mathbf{x})=0 ; h_{i}(\mathbf{x})<0\right. \\ & t=1, \ldots, m<n ; i=1, \ldots, r\end{cases}
$$

em que: $\mathbf{x}=\left(x_{1}, \ldots, x_{j}, \ldots, x_{n}\right)^{T}$ é um vetor de um conjunto compacto $X \subset \mathbb{R}^{n}, f(\mathbf{x})$ é a função objetivo, $g_{t}(\mathbf{x})=0, t=1, \ldots, m$ é uma restrição de igualdade e $h_{i}(\mathbf{x})<0, i=1, \ldots, r$ é uma restrição de desigualdade. Consideramos que $f, g_{t}$ e $h_{i}$ são funções de classe $C^{2}$ e a condição de qualificação de restrições são de (Mangasarian \& Fromovitz, 1976). Ao acrescentarmos as componentes do vetor de variáveis de folga $\mathbf{z}_{\mathbf{1}}=\left(z_{1_{1}}, \ldots, z_{1_{i}}, \ldots, z_{1_{r}}\right)^{T} \in \mathbb{R}_{+}^{r}$ sobre cada restrição de desigualdade de (1) temos:

$$
\begin{cases}\text { Min } & f(\mathbf{x}) \\
\text { s.a: } & \left\{\begin{array}{l}
g_{t}(\mathbf{x})=0 ; h_{i}(\mathbf{x})+z_{1_{i}}=0 ; \\
-z_{1_{i}}<0
\end{array}\right. \\
& t=1, \ldots, m<n ; \quad i=1, \ldots, r\end{cases}
$$

Utilizamos a função barreira logarítmica clássica/modificada $\Psi($.) com estimadores dos multiplicadores de Lagrange sobre as variáveis de folga e apli- 
camos o problema lagrangiano sobre as restrições de igualdade de (2). Assim, o problema (1) é transformado em um problema irrestrito equivalente definido pela função lagrangiana aumentada interior barreira logarítmica clássica/modificada com estimadores dos multiplicadores de Lagrange proposta em (3).

$$
\left\{\begin{array}{l}
\text { Min } \\
L(\boldsymbol{\omega})=f(\mathbf{x})-\mu \sum_{i=1}^{r} \delta_{1_{i}} \Psi\left(z_{1_{i}}\right)-\sum_{t=1}^{m} \lambda_{0_{t}} t_{0_{t}}-\sum_{i=1}^{r} \lambda_{1_{i}} t_{1_{i}} \\
\boldsymbol{\omega}=\left(\mathbf{x}, \mathbf{z}_{\mathbf{1}}, \lambda_{\mathbf{0}}, \lambda_{\mathbf{1}}\right)^{T} \in \mathbb{R}^{n} \times \mathbb{R}^{m} \times \mathbb{R}_{+}^{r} \times \mathbb{R}_{+}^{r}
\end{array}\right.
$$

em que $\mu \in \mathbb{R}_{+}^{*}$ é o parâmetro de barreira; as componentes do vetor $\boldsymbol{\delta}_{1}=\left(\delta_{1_{1}}, \ldots, \delta_{1_{i}}, \ldots, \delta_{1_{r}}\right)^{T} \in \mathbb{R}_{+}^{r}$ são estimadores dos multiplicadores de Lagrange; $-g_{t}(\mathbf{x})$ é uma componente do vetor $\mathbf{t}_{\mathbf{0}}=-\mathbf{g}(\mathbf{x})=\left(-g_{t}(\mathbf{x})\right)^{T}, t=1, \ldots, m \quad ; \quad-\left(h_{i}(\mathbf{x})+z_{1_{i}}\right)$ é uma componente do vetor $\mathbf{t}_{\mathbf{1}}=-\left(\mathbf{h}(\mathbf{x})+\mathbf{z}_{\mathbf{1}}\right)=\left(-\left(h_{i}(\mathbf{x})+z_{1_{i}}\right)\right)^{T}, i=1, \ldots, r ;$

$\lambda_{0}=\left(\lambda_{0_{1}}, \ldots, \lambda_{0_{m}}\right)^{T} \in \mathbb{R}^{m}$ é o vetor de multiplicadores de Lagrange referente à restrição de igualdade $\mathbf{t}_{\mathbf{0}}=\mathbf{0}$ $; \lambda_{1}=\left(\lambda_{1_{1}}, \ldots, \lambda_{r_{r}}\right)^{T} \in \mathbb{R}_{+}^{r}$ é o vetor de multiplicadores de Lagrange referente às restrições de igualdade $\mathbf{t}_{\mathbf{1}}=\mathbf{0}$ e; dado o parâmetro de proximidade à fronteira da região de viabilidade primal original do problema (2) $\tau \in \mathbb{R}_{+}^{*}$, definimos:

$$
\psi\left(z_{i}\right)=\left\{\begin{array}{l}
\Psi_{1}\left(z_{1_{i}}\right)=\ln \left(z_{1_{i}}\right), \text { se } z_{1_{i}}>\tau \\
\Psi_{2}\left(z_{1_{i}}\right)=\ln \left(\left(\mu+z_{1_{i}}\right) / \mu\right), \text { se } z_{1_{i}} \leq \tau
\end{array}\right.
$$

em que $\Psi_{1}\left(z_{1_{i}}\right)$ é a função barreira logarítmica clássica a qual foi definida por (Frisch, 1955) e $\Psi_{2}\left(z_{1_{i}}\right)$ é a função barreira logarítmica modificada a qual foi definida por (Polyak, 1992), logo, a função $\Psi($. está definida em seu domínio $D(\Psi)=\left\{z_{1_{i}} \in \mathbb{R} ; z_{1_{i}}>0 \text { ou }-\mu<z_{1_{i}} \leq 0\right\}^{2}$.

As condições de KKT são obtidas ao aplicarmos a condição necessária de otimalidade . Essas condições são apresentadas em (5):

$\nabla L(\boldsymbol{\omega})=\left\{-\mathbf{m}=\mathbf{0} ;-\mathbf{t}_{\mathbf{0}}=\mathbf{0} ;-\mathbf{t}_{\mathbf{1}}=\mathbf{0} ; \bar{Z}_{1} \boldsymbol{\lambda}_{1}-\mu \boldsymbol{\delta}_{1}=\mathbf{0}\right\}(5)$
em $\quad \boldsymbol{\omega}=\left(\mathbf{x}, \mathbf{z}_{1}, \boldsymbol{\lambda}_{\mathbf{0}}, \boldsymbol{\lambda}_{1}\right)^{T} ;$
$-\mathbf{m}=\nabla f(\mathbf{x})+\nabla \mathbf{g}(\mathbf{x})^{\mathrm{T}} \boldsymbol{\lambda}_{\mathbf{0}}+\nabla \mathbf{h}(\mathbf{x})^{\mathrm{T}} \boldsymbol{\lambda}_{\mathbf{1}}$ é o vetor residual da condição de viabilidade dual; $\nabla \mathbf{g}(\mathbf{x})=\left(\nabla g_{1}(\mathbf{x})^{T}, \ldots, \nabla g_{m}(\mathbf{x})^{T}\right)^{T} \in \mathbb{R}^{m \times n}$ e

${ }^{1}$ Neste trabalho, definimos $\tau=10^{-4}$ fixo.

${ }^{2}$ Definimos por região de viabilidade primal relaxada ao conjunto $\left\{z_{1_{i}} \in \mathbb{R} ;-\mu<z_{1_{i}} \leq 0\right\}$. $\nabla \mathbf{h}(\mathbf{x})=\left(\nabla h_{1}(\mathbf{x})^{T}, \ldots, \nabla h_{r}(\mathbf{x})^{T}\right)^{T} \in \mathbb{R}^{r \times n} \quad$ são, respectivamente, as matrizes jacobianas dos funcionais $\mathbf{g}(\mathbf{x})=\left(g_{1}(\mathbf{x}), \ldots, g_{m}(\mathbf{x})\right)^{T}$ e $\mathbf{h}(\mathbf{x})=\left(h_{1}(\mathbf{x}), \ldots, h_{r}(\mathbf{x})\right)^{T} ;$ e a componente diagonal da matriz $\bar{Z}_{1}^{-1}$ é $1 / z_{1_{i}}$ se $z_{1_{i}}>\tau$ ou $1 /\left(\mu+z_{1_{1}}\right)$ se $z_{1_{i}} \leq \tau$.

Descrevemos o método previsor-corretor primal-dual barreira logarítmica clássica/modificada (MPIBLC/M) a partir da linearização de cada equação do sistema (5) por um polinômio de Taylor de primeira ordem e determinamos o vetor incógnita de direções de busca $\mathbf{d}_{\boldsymbol{\omega}}=\left(\mathbf{d}_{\mathbf{x}}, \mathbf{d}_{\mathbf{z}_{1}}, \mathbf{d}_{\lambda_{0}}, \mathbf{d}_{\lambda_{1}}\right)^{T}$. O MPIBLC/M é um método que gera uma sequência de pontos $\omega^{\mathbf{k}}, k \in \mathbb{N}$ a partir de um ponto inicial $\omega^{0}$ viável ao problema $(3)^{3}$. Considerando $\omega^{\mathbf{k}}$ e a linearização por Taylor do sistema $\nabla L\left(\boldsymbol{\omega}^{\mathbf{k}}\right)=\mathbf{0}$, obtemos o sistema linear expresso em (6):

$$
A_{k} \mathbf{d}_{\omega}^{\mathbf{k}}=\mathbf{b}^{\mathbf{k}}
$$

em que:

$$
A_{k}=\left(\begin{array}{cccc}
\mathrm{K} & 0 & \nabla \mathbf{g}\left(\mathbf{x}^{\mathbf{k}}\right)^{T} & \nabla \mathbf{h}\left(\mathbf{x}^{\mathbf{k}}\right)^{T} \\
\nabla \mathbf{g}\left(\mathbf{x}^{\mathbf{k}}\right) & 0 & 0 & 0 \\
\nabla \mathbf{h}\left(\mathbf{x}^{\mathbf{k}}\right) & I_{r} & 0 & 0 \\
0 & \Lambda_{1_{k}} & 0 & Z_{1_{k}}
\end{array}\right)
$$

é a matriz jacobiana do sistema (5); $\mathrm{K}=\nabla^{2} f\left(\mathbf{x}^{\mathbf{k}}\right)+\sum_{t=1}^{m}\left[\lambda_{0_{t}}^{k} \nabla^{2} g_{t}\left(\mathbf{x}^{\mathbf{k}}\right)\right]+\sum_{i=1}^{r}\left[\lambda_{1_{i}}^{k} \nabla^{2} h_{i}\left(\mathbf{x}^{\mathbf{k}}\right)\right] ;$ $I_{r}$ é a matriz identidade de ordem $r$ e $\Lambda_{1_{k}}=\operatorname{diag}\left(\lambda_{1_{1}}^{k}, \ldots, \lambda_{1_{i}}^{k}, \ldots, \lambda_{1_{r}}^{k}\right) ; \mathbf{d}_{\mathbf{\omega}}^{\mathbf{k}}=\left(\mathbf{d}_{\mathbf{x}}^{\mathbf{k}}, \mathbf{d}_{\mathbf{z}_{1}}^{\mathbf{k}}, \mathbf{d}_{\lambda_{0}}^{\mathbf{k}}, \mathbf{d}_{\lambda_{1}}^{\mathbf{k}}\right)^{T} \mathrm{e}$ $\mathbf{b}^{\mathbf{k}}$ é o vetor residual definido por:

$$
\mathbf{b}^{\mathbf{k}}=\left(\mathbf{m}^{\mathbf{k}}, \mathbf{t}_{\mathbf{0}}^{\mathbf{k}}, \mathbf{t}_{\mathbf{1}}^{\mathbf{k}},-Z_{1_{k}} \lambda_{\mathbf{1}}^{\mathbf{k}}+\mu_{k} \mathbf{\delta}_{\mathbf{1}}^{\mathbf{k}}-D_{z_{1}^{k}} \mathbf{d}_{\lambda_{1}}^{\mathbf{k}}\right)^{T}(7)
$$

em que:

$$
D_{z_{1}^{k}}=\operatorname{diag}\left(d_{z_{1_{1}}}^{k}, \ldots, d_{z_{1_{i}}}^{k}, \ldots, d_{z_{1_{r}}}^{k}\right) \text {. }
$$

Para a definição do procedimento previsor, desprezamos o resíduo não-linear $D_{z_{1}^{k}} \mathbf{d}_{\lambda_{1}}^{\mathbf{k}}$, isto é, a priori definimos esse resíduo como nulo, pois desconhecemos essas direções de busca e consideramos $\mu_{k} \neq 0$ no cálculo das direções de busca primal e dual, assim este método é uma variante dos métodos apresentados em (Mehrotra, 1992) e (Wu, et al., 1994). Uma vez calculadas as direções de busca primais e duais através da expressão (9), as direções de busca são corrigidas, agora, considerando o resíduo $D_{z_{1}^{k}} \mathbf{d}_{\lambda_{1}}^{\mathbf{k}}$ como não-nulo utilizando-se também (9). Assim,

\footnotetext{
3 Note que a região de viabilidade primal relaxada $0 \leq z_{1_{i}}^{0}<\mu_{0}$ é interessante, pois permite-nos escolhas $\mathbf{x}^{0}$ tais que $h_{i}\left(\mathbf{x}^{0}\right) \geq 0<\mu_{0}$, principalmente se há muitas restrições não-lineares e não-convexas.
} 
com procedimentos algébricos, definimos, a seguir, as equações que definem o cálculo das direções primais e duais do procedimento previsor e corretor:

$$
\begin{aligned}
& \mathbf{d}_{\lambda_{0}}^{\mathbf{k}}=-\left(\nabla \mathbf{g}\left(\mathbf{x}^{\mathbf{k}}\right) \theta_{k}^{-1} \nabla \mathbf{g}\left(\mathbf{x}^{\mathbf{k}}\right)^{\mathbf{T}}\right)^{-1}\left(\nabla \mathbf{g}\left(\mathbf{x}^{\mathbf{k}}\right) \theta_{k}^{-1} \mathbf{r}_{\mu}^{\mathbf{k}}+\mathbf{t}_{\mathbf{0}}^{\mathbf{k}}\right)- \\
& -\lambda_{0}^{\mathbf{k}} \\
& \mathbf{d}_{\mathbf{x}}^{\mathbf{k}}=-\theta_{k}^{-1}\left(\mathbf{r}_{\mu}^{\mathbf{k}}+\nabla \mathbf{g}\left(\mathbf{x}^{\mathbf{k}}\right)^{\mathbf{T}}\left(\lambda_{0}^{\mathbf{k}}+\mathbf{d}_{\lambda_{0}}^{\mathbf{k}}\right)\right) \\
& \mathbf{d}_{\mathrm{z}_{1}}^{\mathrm{k}}=-\nabla \mathbf{h}\left(\mathbf{x}^{\mathrm{k}}\right) \mathbf{d}_{\mathrm{x}}^{\mathrm{k}}+\mathbf{t}_{1}^{\mathrm{k}} \\
& \mathbf{d}_{\lambda_{1}}^{\mathbf{k}}=-Z_{1_{k}}^{-1} \Lambda_{1_{k}} \mathbf{d}_{\mathbf{z}_{1}}^{\mathbf{k}}+\mu_{k} Z_{1_{k}}^{-1} \delta_{1}^{\mathbf{k}}-\lambda_{1}^{\mathbf{k}}+\mathbf{v} ; \\
& \mathbf{r}_{\mu}^{\mathbf{k}}=\nabla f\left(\mathbf{x}^{\mathbf{k}}\right)+\mathbf{c}^{\mathbf{k}}+\mu_{k} \varphi^{\mathbf{k}}
\end{aligned}
$$

onde:

$$
\theta_{k}=\mathrm{K}+\nabla \mathbf{h}\left(\mathbf{x}^{\mathbf{k}}\right)^{\mathbf{T}} Z_{1_{k}}^{-1} \Lambda_{1_{k}} \nabla \mathbf{h}\left(\mathbf{x}^{\mathbf{k}}\right)
$$

é a matriz hessiana da função lagrangiana $L\left(\omega^{\mathbf{k}}\right)$;

$$
\boldsymbol{v}=\left\{\begin{array}{l}
\mathbf{0} \in \mathbb{R}^{r}, \\
\text { se o procedimento previsor está em operação } \\
-Z_{1_{k}}^{-1} D_{z_{1}^{k}} \mathbf{d}_{\lambda_{1}}^{\mathbf{k}}, \\
\text { se o procedimento corretor está em operação }
\end{array}\right.
$$

$$
\mathbf{c}^{\mathbf{k}}=\left\{\begin{array}{l}
-\nabla \mathbf{h}\left(\mathbf{x}^{\mathbf{k}}\right)^{\mathbf{T}} Z_{1_{k}}^{-1} \Lambda_{1_{k}} \mathbf{t}_{1}^{\mathbf{k}}, \\
\text { se o procedimento previsor está em operação } \\
-\nabla \mathbf{h}\left(\mathbf{x}^{\mathbf{k}}\right)^{\mathbf{T}}\left(Z_{1_{k}}^{-1} \Lambda_{1_{k}} \mathbf{t}_{1}^{\mathbf{k}}+Z_{1_{k}}^{-1} D_{z_{1}^{k}} \mathbf{d}_{\lambda_{1}}^{\mathbf{k}}\right), \\
\text { se o procedimento corretor está em operação }
\end{array}\right.
$$

$$
\boldsymbol{\varphi}^{\mathbf{k}}=\nabla \mathbf{h}\left(\mathbf{x}^{\mathbf{k}}\right)^{\mathbf{T}} Z_{1_{k}}^{-1} \boldsymbol{\delta}_{1}^{\mathbf{k}} .
$$

Definimos por: $\tilde{\mathbf{d}}_{\omega}^{\mathbf{k}}=\left(\tilde{\mathbf{d}}_{\mathbf{x}}^{\mathbf{k}}, \tilde{\mathbf{d}}_{\mathbf{z}_{1}}^{\mathbf{k}}, \tilde{\mathbf{d}}_{\lambda_{0}}^{\mathbf{k}}, \tilde{\mathbf{d}}_{\lambda_{1}}^{\mathbf{k}}\right)^{T}$ o vetor de direções de busca obtidas pelo procedimento corretor do MPIBLC/M. A estratégia de passo longo (PL), apresentada em (14), é definida pelo cálculo do tamanho do passo longo primal $\bar{\alpha}_{p}$ e do tamanho do passo longo dual $\bar{\alpha}_{d}$ aplicados sobre a direção $\tilde{\mathbf{d}}_{\omega}^{\mathbf{k}}$. A PL é uma variante da estratégia apresentada em (Granville, 1994) a qual é descrita em (Pinheiro, 2012).

$$
\begin{aligned}
& \bar{\alpha}_{p}=1,005 \times \min \{\underbrace{\min }_{z_{i_{i}}^{k}>0 \mathrm{e} \widetilde{d} z_{i_{i}^{k}}^{k}<0}\left\{-\frac{z_{1_{i}}^{k}}{\widetilde{d}_{z_{1_{i}}}^{k}}\right\}, 1\} \\
& \bar{\alpha}_{d}=1,005 \times \min \{\underbrace{\min }_{\lambda_{i_{i}}^{k}>0 \mathrm{e}\left(\tilde{d}_{\lambda_{i}}^{k}\right)_{i}<0}\left\{-\frac{\lambda_{1_{i}}^{k}}{\tilde{d}_{\lambda_{1_{i}}}^{k}}\right\}, 1\} .
\end{aligned}
$$

Destacamos que o valor empírico 1,005 é maior que o valor 0,9995 , definido pela maioria dos autores que utilizam o método primal-dual em seus trabalhos, pois estes autores trabalham somente com função barreira logarítmica clássica. Porém, o valor 1,005 permite ao MPIBLC/M-PL explorar pontos $-\mu_{k}<z_{1_{i}}^{k} \leq 0$, isto é, pontos $z_{1_{i}}^{k}$ que estão na região de viabilidade primal relaxada. Atualizamos o vetor $\boldsymbol{\omega}^{\mathbf{k}+1}$ da seguinte maneira:

$$
\begin{aligned}
& \omega^{\mathbf{k}+1}=\left(\mathbf{x}^{\mathbf{k}+1}, \mathbf{z}_{1}^{\mathbf{k}+1}, \lambda_{0}^{\mathbf{k}+1}, \lambda_{1}^{\mathbf{k}+1}\right)^{T}= \\
& =\left(\mathbf{x}^{\mathbf{k}}, \mathbf{z}_{1}^{\mathbf{k}}, \lambda_{0}^{\mathbf{k}}, \lambda_{1}^{\mathbf{k}}\right)^{T}+\left(\bar{\alpha}_{p} \tilde{\mathbf{d}}_{\mathbf{x}}^{\mathbf{k}}, \bar{\alpha}_{p} \tilde{\mathbf{d}}_{\mathbf{z}_{1}}^{\mathbf{k}}, \tilde{\mathbf{d}}_{\lambda_{0}}^{\mathbf{k}}, \bar{\alpha}_{d} \tilde{\mathbf{d}}_{\lambda_{1}}^{\mathbf{k}}\right)^{T} .
\end{aligned}
$$

A atualização dos estimadores dos multiplicadores de Lagrange $\boldsymbol{\delta}_{\mathbf{i}}^{\mathbf{k}}$ é adotada como uma generalização da regra proposta em (Polyak, 1992) dada em (16):

$$
\delta_{1_{i}}^{k+1}=\left(\delta_{1_{i}}^{k} \mu_{k}\right) /\left(\mu_{k}+z_{1_{i}}^{k}\right)
$$

e é apresentada, de acordo com (Pinheiro, 2012), em (17):

$$
\delta_{1}^{k+1}=\lambda_{1}^{k+1}
$$

em que $\lambda_{1}^{k+1}$ está definido em (15). A atualização apresentada em (17) permite que a aplicação integrada entre $\Psi_{1}\left(z_{1_{i}}\right)$ e $\Psi_{2}\left(z_{1_{i}}\right)$ seja estável e robusto, pois $\delta_{1_{i}}^{k+1}$ contém informações as quais contemplam as restrições de igualdade e de desigualdade de (1). $\mathrm{O}$ parâmetro de barreira $\mu_{k+1}$ é atualizado conforme a seguinte heurística:

$$
\mu_{k+1}=0,25 \mu_{k}
$$

Após essa atualização, realizamos o seguinte teste: se $z_{1_{i}}^{k+1} \geq-\mu_{k+1}, \forall i=1, \ldots, r$, então a heurística (18) permanece; caso contrário, $\mu_{k+1}$ recebe a atualização, mostrada em (19):

$$
\mu_{k+1}=-(1,25) \min \left(\mathbf{z}_{\mathbf{1}}^{\mathbf{k}}\right)
$$

O sinal "-" em (19) é devido ao fato de o sinal de $z_{1_{i}}^{k}$ não pertencer a $D(\Psi)$.

A estratégia de convergência global (CG) proposta é baseada em uma variante daquelas apresentadas em (Benson, et al., 2000) e (Bazaraa, et al., 2006). Esta estratégia consiste nos seguintes procedimentos:

Procedimento 1: Após a construção da matriz $\theta_{k}$ dada em (10), utilizamos (20) para fazer uma perturbação diagonal sobre esta matriz:

$$
\theta_{k} \leftarrow \theta_{k}+\beta_{k} I_{n}
$$

em que $\beta_{k}>0$ é denominado de parâmetro de amortecimento do método de Levenberg-Marquardt (Levenberg, 1944) (Marquardt, 1963). Um valor $\beta_{0}>0$ inicial suficientemente pequeno deve ser fornecido.

Procedimento 2: A matriz $\theta_{k}$ é submetida à Decomposição de Cholesky (DC), para ao cálculo da matriz triangular inferior $G_{k}$, de modo que $\theta_{k}=G_{k} G_{k}^{T}$. Durante o processo de DC, enquanto pelo menos um dos elementos $G_{k}(j, j)=\sqrt{\theta_{k}(j, j)-\sum_{o=1}^{j-1} G_{k}^{2}(j, o)} ; j=1, \cdots, n$ não for um número real atualizamos $\beta_{k}$ conforme heurística descrita em (21) e, em seguida, atualizamos a matriz $\theta_{k}$, conforme (20): 


$$
\beta_{k} \leftarrow \beta_{k}\left(1+\sqrt{(\sqrt{5}-1)^{2} 0,25^{2}+1}\right) / 2 .
$$

De acordo com (Pinheiro, 2012), o parâmetro $\beta_{k}$ influencia tanto na direção quanto no tamanho do passo, isto é, na trajetória dos pontos $\omega^{\mathbf{k}}$ e também infere diretamente sobre o condicionamento de $\theta_{k}$ tornando o método mais estável durante os ciclos iterativos e/ou se algum $z_{1_{i}}^{k} \rightarrow 0$. Para a inicialização de um novo ciclo $k+1$ do MPIBLC/M-PLCG atualizamos $\beta_{k+1}$ conforme estratégia adaptada de (Bazaraa, et al., 2006), descrita a seguir.

i) Se $L\left(\boldsymbol{\omega}^{\mathbf{k}}\right)-L\left(\boldsymbol{\omega}^{\mathbf{k}+1}\right)<0,25$, então $\beta_{k+1}$ é atualizado pela heurística proposta em (22):

$$
\beta_{k+1}=4 \beta_{k} /\left((\sqrt{5}+1)+\sqrt{16 \times 0,25^{2}+(\sqrt{5}+1)^{2}}\right)(22)
$$

ii) Se $L\left(\omega^{\mathbf{k}}\right)-L\left(\boldsymbol{\omega}^{\mathbf{k}+1}\right)>0,75$, então $\beta_{k+1}$ é atualizado pela heurística (23):

$$
\beta_{k+1}=\beta_{k}\left(1+\sqrt{(\sqrt{5}-1)^{2} 0,25^{2}+1}\right) / 2 ;
$$

iii) $\quad$ Se $\quad 0,25 \leq L\left(\omega^{\mathbf{k}}\right)-L\left(\omega^{\mathbf{k}+1}\right) \leq 0,75$, então $\beta_{k+1}=\beta_{k}$.

\section{O MPIBLC/M-PLCG aplicado ao FPOR e Re- sultados Numéricos}

O modelo, na variável $\mathbf{x}=(\mathbf{V}, \boldsymbol{\gamma})^{T}$, de minimização das perdas de potência ativa na transmissão para o FPOR é definido por:

$$
\left\{\begin{array}{c}
\quad \text { Min } f(\mathbf{x})=\sum_{i=1}^{N L} g_{k m_{i}}\left(V_{k}^{2}+V_{m}^{2}-2 V_{k} V_{m} \cos \left(\gamma_{k m}\right)\right) \\
s a: \begin{array}{c}
\Delta P_{k}=0 \\
\Delta Q_{k}=0
\end{array} \\
Q_{k}^{\min } \leq V_{k} \sum_{m \in \Omega} V_{m}\left(G_{k m} \operatorname{sen}\left(\gamma_{k m}\right)-B_{k m} \cos \left(\gamma_{k m}\right)\right) \leq Q_{k}^{\max } \\
0,95 \leq V_{k} \leq 1,1
\end{array}\right.
$$

No problema apresentado imediatamente acima, a equação

$\Delta P_{k}=P_{k}^{e s p}-V_{k} \sum_{m \in \Omega} V_{m}\left(G_{k m} \cos \left(\gamma_{k m}\right)+B_{k m} \operatorname{sen}\left(\gamma_{k m}\right)\right) \quad$ é

determinada para todas as barras $k$, com exceção da barra de referência; a equação $\Delta Q_{k}=Q_{k}^{e s p}-V_{k} \sum_{m \in \Omega} V_{m}\left(G_{k m} \operatorname{sen}\left(\gamma_{k m}\right)-B_{k m} \cos \left(\gamma_{k m}\right)\right) \quad$ é

determinada para as barras $k$ de carga, as restrições de desigualdade canalizada é determinada para as barras $k$ com magnitude de tensão controlável; $N L$ é o número de ramos da transmissão; $g_{k m_{i}}$ é a condutância do ramo $i$ que conecta as barras $k$ e $m$; $P_{k}^{e s p}, Q_{k}^{e s p}$ são, respectivamente, as potências ativa e reativa líquidas especificadas para a barra $k ; \Omega$ é o conjunto das barras vizinhas à barra $k$, incluindo-se a própria barra $k$, e $G_{k m}, B_{k m}$ são, respectivamente, as partes real e imaginária do elemento $Y_{k m}$ da matriz admitância de barra.

O MPIBLC/M-PLCG foi implementado em MATLAB 6.1 e foi aplicado aos sistemas elétricos de 3, 9, IEEE-14, IEEE-30, IEEE-57, IEEE-118, IEEE162 e IEEE-300 ${ }^{4}$ barras. Em todos os casos estudados, definimos $\mathbf{V}^{\mathbf{0}}=\mathbf{1} ; \boldsymbol{\gamma}^{\mathbf{0}}=\mathbf{0} ; \mu_{0}=0,05 ; \beta_{0}=0,1$, $\delta_{1_{i}}^{0}=0,1, i=1, \ldots, r$ os limitantes para o controle de reativos são originais do banco de dados e o critério de parada para o método foi de $\left\|\mathbf{b}^{\mathbf{k}}\right\|_{\infty} \leq 10^{-10}$ para todos os sistemas elétricos exceto para o sistema IEEE-300, o qual utilizamos $\left\|\mathbf{b}^{\mathbf{k}}\right\|_{\infty} \leq 10^{-8}$. Medimos o condicionamento da matriz $\theta_{k}$ antes da DC (CADC)

\begin{tabular}{|c|c|c|}
\hline Sistema Elétrico & Iterações & $\mu_{k}$ final \\
\hline 3 barras & 9 & $7.6294 \mathrm{e}-07$ \\
\hline 9 barras & 10 & $1.9073 \mathrm{e}-07$ \\
\hline IEEE-14 & 10 & $2.6412 \mathrm{e}-07$ \\
\hline IEEE-30 & 9 & $7.6294 \mathrm{e}-07$ \\
\hline IEEE-57 & 12 & $1.1539 \mathrm{e}-07$ \\
\hline IEEE-118 & 13 & $1.5044 \mathrm{e}-07$ \\
\hline IEEE-162 & 11 & $4.7684 \mathrm{e}-08$ \\
\hline IEEE-300 & 17 & $8.8842 \mathrm{e}-09$ \\
\hline Sistema Elétrico & $\left\|\mathbf{b}^{\mathbf{k}}\right\|_{\infty}$ final & CADC \\
\hline 3 barras & $5.6748 \mathrm{e}-12$ & $1.3022 \mathrm{e}+06$ \\
\hline 9 barras & $2.1748 \mathrm{e}-12$ & $3.7842 \mathrm{e}+20$ \\
\hline IEEE-14 & $1.4690 \mathrm{e}-11$ & $4.0224 \mathrm{e}+08$ \\
\hline IEEE-30 & $6.1297 \mathrm{e}-11$ & $8.1641 \mathrm{e}+07$ \\
\hline IEEE-57 & $1.3926 \mathrm{e}-12$ & $5.2473 e+09$ \\
\hline IEEE-118 & $1.4431 \mathrm{e}-12$ & $1.5047 \mathrm{e}+10$ \\
\hline IEEE-162 & $8.9381 \mathrm{e}-11$ & $1.3602 \mathrm{e}+21$ \\
\hline IEEE-300 & $1.1695 \mathrm{e}-09$ & $3.8725 \mathrm{e}+24$ \\
\hline Sistema Elétrico & CDDC & $\begin{array}{l}\text { Perdas } \\
\text { Megawatts }\end{array}$ \\
\hline 3 barras & $1.2500 \mathrm{e}+06$ & 12,91584094822280 \\
\hline 9 barras & $1.2785 \mathrm{e}+10$ & 4,11369184655652 \\
\hline IEEE-14 & $2.6924 \mathrm{e}+08$ & 12,29251166783213 \\
\hline IEEE-30 & $6.0050 e+07$ & 16,08931485721277 \\
\hline IEEE-57 & $5.1933 e+09$ & 22,97598670741661 \\
\hline IEEE-118 & $1.4768 \mathrm{e}+10$ & 107,7019055917016 \\
\hline IEEE-162 & $1.3028 \mathrm{e}+10$ & 146,1992165567937 \\
\hline IEEE-300 & $2.1869 \mathrm{e}+14$ & 351,4749499225414 \\
\hline
\end{tabular}
e depois da DC (CDDC) e apresentamos os resultados apenas da última iteração. Para a aplicação do método proposto os taps dos transformadores foram considerados constantes. A Tabela 1 nos apresenta os principais resultados obtidos:

Tabela 1- Solução Geral do FPOR e principais resultados obtidos pelo MPIBLC/M-PLCG.

${ }^{4} \mathrm{O}$ transformador defasador relacionado ao ramo 196-2040 foi substituído por um transformador em fase. 


\section{Conclusões}

Neste trabalho apresentamos o método previsorcorretor primal-dual de pontos interiores barreira logarítmica clássica/modificada com estratégias de passo longo e de convergência global (MPIBLMC/M-PLGC), o qual foi aplicado ao problema de fluxo de potência ótimo reativo (FPOR) com taps dos transformadores constantes. Neste trabalho foram apresentadas as principais características do método proposto tais como: é variante dos tradicionais métodos previsor-corretor primal-dual de pontos interiores; o método propõe uma robusta aplicação integrada entre os métodos de barreira clássica e barreira modificada; nova proposta de atualização dos estimadores dos multiplicadores de Lagrange, a qual contempla informações de restrições de igualdade e de desigualdade; a aplicação das estratégias de passo longo, correção de trajetória, aumento do condicionamento da matriz hessiana e de convergência global e uma heurística de atualização do parâmetro de barreira contribuíram significativamente no fortalecimento do método, possibilitando a esse a resolução de problemas de programação não-linear em domínio não-convexo, tais como o FPOR. Os resultados quantitativos mostraram que o método é robusto, pois foi capaz de obter solução ótima primal e dual em todos os sistemas elétricos estudados com um número relativamente baixo de iterações e com alta precisão, de acordo com a Tabela 1 apresentada.)

\section{Agradecimentos}

Este trabalho contou com a graça de Deus e com apoio da Capes através do programa de mestrado em Engenharia Elétrica da UNESP em Bauru.

\section{Referências Bibliográficas}

Bazaraa, M. S., Sherali, H. D. \& Shetty, C. M., 2006. Nonlinear Progremming: Theory and Algorithms. 3 ed. New Jersey: Wiley Interscience.

Benson, H. Y., Shanno, D. F. \& Vanderbei, R. J., 2000. Iinterior-Ponit Methods for noncovex nonlinear programming: Jamming and comparative numerical testeing. Operations Research and Financial Engineering.

Frisch, K. R., 1955. The Logarithmic Potential Method for Convex Programming.

Granville, S., 1994. Optimal Reactive Dispatch Through Interior Point Methods. IEEE Transactions on Power Systems, Volume 9, pp. 136-146. DOI: $10.1109 / 59.317548$

Levenberg, K., 1944. A Method for the Solution of Certain Non-Linear Problems in Least Squares. Quarterly of Applied Mathematics, pp. 164-168.

Mangasarian, O. L. \& Fromovitz, S., 1976. The Fritz-John necessary optimality conditions in the Presence of Equality and Inequality Constraints.
Journal of mathematical analysis and applications, Volume 17, pp. 37-47.

Marquardt, D., 1963. An Algorithm for LeastSquares Estimation of Nonlinear Parameters. SIAM Journal on Applied Mathematics, pp. 431-441.

Mehrotra, S., 1992. On implementation of a primal-dual interior point method. SIAM Journal on Optimization, Volume 2, pp. 572-601.

Pinheiro, R. B. N., 2012. Um método previsorcorretor primal-dual de pontos interiores barreira logarítmica modificada, com estratégias de global e de ajuste cúbico, para problemas de programação não-linear e não-convexa., Bauru: Programa de PósGraduação em Engenharia Elétrica (Dissertação de Mestrado), FEB/UNESP.

Polyak, R., 1992. Modified barrier functions. Mathematical Programming, v.54(2), p. 177 - 222.

Wu, Y., Debs, A. S. \& Marsten, R. E., 1994. A Direct Nonlinear Predictor- Corrector Primal-Dual Interior Point Algorithm for Optimal Power Flow. IEEE Transactions on Power Systems, Volume 9, pp. 876-883. 